\title{
MINI-REVIEW
}

\section{Potentials of interleukin-27 (IL-27) as an immunotherapeutic cytokine in cancer therapy}

\author{
Wei Boon Yap ${ }^{1 *}$, Shaktypreya Nadarajah ${ }^{1}$, Nadiah Shidik ${ }^{1}$, \\ Noorjahan Banu Mohammed Alitheen ${ }^{2}$ \\ ${ }^{1}$ Program of Biomedical Sciences, School of Diagnostic and Applied Health Sciences, Faculty of Health Sciences, \\ Universiti Kebangsaan Malaysia, Kuala Lumpur, Malaysia \\ ${ }^{2}$ Department of Cell and Molecular Biology, Faculty of Biotechnology and Biomolecular Sciences, Universiti Putra \\ Malaysia, UPM Serdang, Selangor, Darul Ehsan, Malaysia
}

\begin{abstract}
Cancer immunotherapy using cytokines has been sought as an alternative therapeutic approach for treating cancers. Besides remarkable immunoregulatory properties, interleukin (IL)-27 has recently been shown to possess promising anticancer functions; hence, its potential roles in cancer immunotherapy. Although proven to be effective against cancer cell growth and angiogenesis, given its dual immune-regulating functions (pro-inflammatory and anti-inflammatory), the use of IL-27 as a cancer immunotherapeutic cytokine could possibly be a two-edged sword without meticulous and thorough research. This mini-review mainly discusses the functions and future prospects of IL-27 as an effective anticancer cytokine. Hopefully, it imparts useful insights into the potential applications of IL-27 in cancer immunotherapy
\end{abstract}

Keywords: interleukins; IL-27; cytokines; immunotherapy; cancers

ARTICLE INFO

Received: July 2, 2019

Accepted: August 2, 2019

Available online: August 15, 2019

*CORRESPONDING AUTHOR

Wei Boon Yap, Program of Biomedical Sciences, School of Diagnostic and

Applied Health Sciences, Faculty of Health Sciences, Universiti

Kebangsaan Malaysia, Jalan Raja Muda Abdul Aziz, 50300 Kuala

Lumpur, Malaysia; yapweiboon@ukm. edu.my

\section{CITATION}

Yap WB, Nadarajah S, Shidik N, et al. Potentials of interleukin-27 (IL-

27) as an immunotherapeutic cytokine in cancer therapy. Trends

Immunother 2019; 3(2): 76-78. doi: 10.24294/ti.v3.i2.121.

\section{COPYRIGHT}

Copyright (C) 2019 by author(s) and EnPress Publisher LLC. This work is licensed under the Creative Commons Attribution-NonCommercial 4.0 International License (CC BY-NC 4.0). http://creativecommons.org/licenses/ by $/ 4.0 /$

\section{Introduction}

Given various detrimental side effects of modern anticancer treatments, cytokine immunotherapy has been sought as an alternative therapeutic approach. A combinatorial immunotherapy of interleukin (IL)-12 with a breast cancer-specific monoclonal antibody, trastuzumab and an anticancer drug namely paclitaxel was found to inhibit breast cancer cell growth as high as $52 \%$ in the first-phase clinical trial ${ }^{[1]}$. However, repeated administration of IL-12 did somehow render toxicity to metastatic kidney cancer and breast cancer patients ${ }^{[2]}$. As a result, to optimize the use of cytokines in cancer immunotherapy, scrupulous research on therapeutic cytokines especially those capable of triggering both pro-inflammatory and anti-inflammatory reactions is undoubtedly entailed. In this mini-review, the anticancer functions of IL27 , with pro- and anti-inflammatory effects, and its future prospects in cancer immunotherapy are highlighted.

\section{Interleukins are more than just immune modulatory cytokines}

Interleukins (ILs) have long been recognized for their immune modulatory functions such as regulating local and systemic immunity by controlling immune cell proliferation, differentiation and motility. By doing so, immune homeostasis is achieved between pro- and anti-inflammatory reactions ${ }^{[3]}$. In the recent decades, the potentials of ILs have been further explored and found effective as immunotherapeutics against various cancers. ILs such as IL-2 has been approved by US Food and Drug Administration (FDA) for use in cancer immunotherapy, for instance in treating metastatic melanoma ${ }^{[4,5]}$. Besides IL-2, IL-12 was also found to exhibit direct inhibition on cancer cell growth while promoting activation of immune cells with cancer killing functions ${ }^{[2]}$. The anticancer properties of IL-12 were well observed in various 
maglinant models such as B16 melanoma, breast ductal adenocarcinoma, C26 colon carcinoma and metastatic lung carcinoma ${ }^{[6]}$.

Although proven with prominent anticancer functions, IL-12 immunotherapy was, however, reported to render adverse side effects such as hypersplenism in melanoma patients ${ }^{[7]}$ which in turn results in extravascular hemolysis and enlargement of spleen. To overcome the adverse effects, IL-27, which is also a member of IL-12 family, has been merited for its antitumor benefits without causing much adverse toxic effects in cancer model ${ }^{[4]}$. Of note, IL-27 could be harnessed as an alternative anticancer cytokine in cancer immunotherapy ${ }^{[8]}$.

IL-27 is composed of Epstein-Barr virus-induced gene-3 (EBI-3) and p28 subunits that interact with cell receptors that are made up of WSX-1 and gp130 subunits ${ }^{[9]}$. IL-27 is often produced by antigen presenting cells and particularly important in T-cell proliferation. IL-27 functions as both pro- and antiinflammatory cytokine, for instance, it promotes Th1 responses but inhibits proliferation of Th17 that is responsible for many inflammatory reactions ${ }^{[10]}$. As a result, the use of IL-27 as a therapeutic agent entails scrupulous and rigorous investigation so it does not produce undesired outcomes in recipients. This is particularly crucial in cancer therapy as any agents that generate excessive inflammation would cause drug resistance and metastasis in cancers ${ }^{[10]}$.

\section{Inhibitory functions of IL-27 on cancer cells}

To better employ IL-27 as an immunotherapeutic cytokine for cancers, the mechanisms of actions on how IL-27 exerts its anticancer functions have to be unraveled. As an immunoregulatory cytokine, IL-27 signals activation of natural killer (NK) and cytotoxic $\mathrm{T}$ cells which possess cancer killing properties $^{[11]}$. Besides regulating antitumor immune responses, IL-27 has also been proven to execute direct inhibitory functions on cancers. For instance, inhibiting pediatric B-acute lymphoblastic leukemia cell proliferation ${ }^{[12]}$, prohibiting angiogenesis in melanoma B16F10 cell line and blocking metastasis of lung cancer in mouse mode ${ }^{[13]}$. Inhibition of metastasis of lung cancer by IL-27 is mostly attributed to suppression of cyclooxygenase-2 $(\mathrm{COX}-2)^{[14]}$ and downregulation of epithelium-mesenchymal transition (EMT) in lung cancer cells ${ }^{[15]}$.
Apart from the aforementioned antitumour activities, cancer cell death via apoptosis is often targetted by many antitumour agents. Antitumour agents with apoptotic properties trigger mitochondrial oxidative stress which then results in the release of cytochrome-C, activation of caspase cascade and eventually cell death ${ }^{[16]}$. Ruiz-Ruiz et $a l .{ }^{[17]}$ showed that IL-27 was able to trigger apoptosis in breast cancer cells by stimulating interferon (IFN)- $\gamma$ expression. Activation of IFN- $\gamma$ subsequently increases the expression of pro-apoptotic protein, caspase- 8 in MCF-7 and MDA-MB-231 breast cancer cell lines. Through caspase- 8 activity, the intrinsic pathway is activated and cytochrome-c is released and interacts with protease activating factor-1 (Apaf-1) which in turn promotes caspase-9 and caspase-3 activation and apoptosis (Figure 1). Collectively, the immunoregulatory, direct inhibitory and apoptosis-promoting functions of IL-27 add up to its potentials as an immunotherapeutic agent.

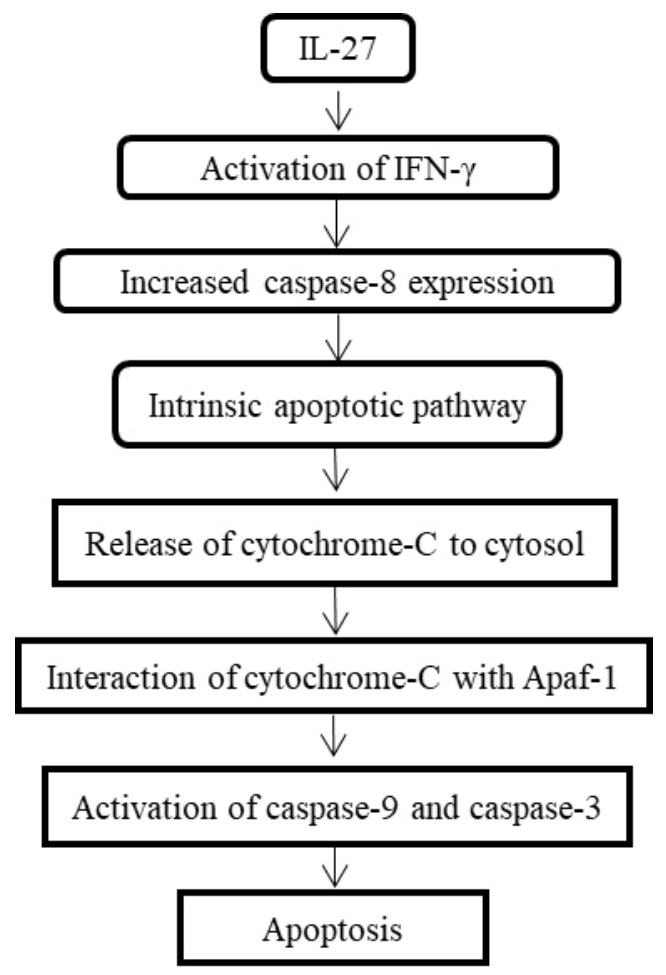

Figure 1. Activation of apoptotic protein cascade by IL27 . Through activation of IFN- $\gamma$, IL-27 is able to induce apoptosis in breast cancer cell lines.

\section{Future prospects of IL-27 in cancer immunotherapy}

IL-27 is unequivocally a prominent immunoregulatory cytokine that promotes both proinflammatory and anti-inflammatory responses. In order to avoid treatment outcomes that could 
encourage metastasis and drug-resistance in cancers which in turn undermine an anticancer therapy regime ${ }^{[10]}$, any claims on the potential use of IL-27 as an immunotherapeutic cytokine should be preceded by meticulous and rigorous experimentations. Anticancer functions of IL-27 can be further explored by (a) detailed investigation on the ability of IL-27 in promoting NK cells and tumor-specific $\mathrm{T}$ cell proliferation ex vivo and how these activated cells target tumors in adoptive cell therapy; (b) expanding the IL-27-induced apoptosis networks in cancer models. With great breadth of understanding on how IL-27 effects anticancer functions in cancer cells, this ensures more precise use of IL-27 as an immunotherapeutic agent without causing much damaging side effects to cancer patients. As a conclusion, IL-27 does hold prominent potentials in cancer immunotherapy. Rigorous empirical evidence would be able to help further unveil its importance in cancer immunotherapy.

\section{Conflict of interest}

The authors declare no potential conflict of interest with respect to the research, authorship, and/ or publication of this article.

\section{Acknowledgments}

The authors thank the Ministry of Higher Education, Malaysia for funding the work under the Fundamental Research Grant Scheme (FRGS/1/2014/SG05/UKM/02/4).

\section{References}

1. Bekaii-Saab TS, Roda JM, Guenterberg KD, et al. A phase I trial of paclitaxel and trastuzumab in combination with interleukin-12 in patients with HER2/neu-expressing malignancies. Mol Cancer Ther 2009; 8(11): 2983-2991. doi: 10.1158/15357163.MCT-09-0820.

2. Tugues S, Burkhard SH, Ohs I, et al. New insights into IL-12-mediated tumor suppression. Cell Death Differ 2015; 22: 237-246. doi: 10.1038/ cdd.2014.134.

3. Zhang JM, An JX. Cytokines, inflammation and pain. Int Anesthesiol Clin 2007; 45(2): 27-37. doi: 10.1097/AIA.0b013e318034194e.

4. Sorensen EW, Gerber SA, Frelinger JG, et al. IL-12 suppresses vascular endothelial growth factor receptor 3 expression on tumor vessels by two distinct IFN- $\gamma$-dependent mechanisms. J
Immunol 2010; 184(4): 1858-1866. doi: 10.4049/ jimmunol.0903210.

5. Lee S, Margolin K. Cytokines in cancer immunotherapy. Cancers 2011; 3: 3856-3893. doi: 10.3390/cancers3043856.

6. Shi X, Liu J, Xiang Z, et al. Gene expression analysis in interleukin-12-induced suppression of mouse mammary carcinoma. Int J Cancer 2004; 110(4): 570-578. doi: 10.1002/ijc.20145.

7. Gollob JA, Mier J, Veenstra K, et al. Phase I trial of twice-weekly intravenous interleukin 12 in patients with metastatic renal cell cancer or malignant melanoma: Ability to maintain IFN- $\gamma$ induction is associated with clinical response. Clinic Cancer Res 2000; 6: 1678-1692.

8. Yoshimoto T, Chiba Y, Furusawa JI, et al. Potential clinical application of interleukin-27 as an antitumor agent. Cancer Sci 2015; 106(9): 11031110. doi: 10.1111/cas.12731.

9. Pflanz S, Timans JC, Cheung J, et al. IL-27, a heterodimeric cytokine composed of EBI3 and p28 protein, induces proliferation of naive $\mathrm{CD}^{+} \mathrm{T}$ cells. Immunity 2002; 16(6):779-790.

10. Li MS, Liu ZZ, Liu JQ, et al. The Yin and Yang aspects of IL-27 in induction of cancer-specific T cell responses and immunotherapy. Immunotherapy 2015; 7(2): 191-200. doi: 10.2217/imt.14.95.

11. Hisada M, Kamiya S, Fujita K, et al. Potent antitumor activity of interleukin-27. Cancer Res 2004; 64: 1152-1156. doi: 10.1158/0008-5472. CAN-03-2084.

12. Canale S, Cocco C, Frasson C, et al. Interleukin-27 inhibits pediatric B-acute lymphoblastic leukemia cell spreading in a preclinical model. Leukemia $2011 ; 25(12)$ : 1815-1824. doi: $10.1038 /$ leu.2011.158.

13. Shimizu M, Shimamura M, Owaki T, et al. Antiangiogenic and antitumor activities of IL-27. J Immunol 2006; 176(12): 7317-7324. doi: 10.4049/ jimmunol.176.12.7317.

14. Ho MY, Leu SJ, Sun GH, et al. IL-27 directly restrains lung tumorigenicity by suppressing cyclooxygenase-2-mediated activities. J Immunol 2009; 183(10): 6217-6226. doi: 10.4049/ jimmunol.0901272.

15. Airoldi I, Tupone MG, Esposito S, et al. Interleukin-27 re-educates intratumoral myeloid cells and down-regulates stemness genes in non-small cell lung cancer. Oncotarget 2015; 6(6): 36943708. doi: 10.18632/oncotarget.2797.

16. Liou GY, Storz P. Reactive oxygen species in cancer. Free Radic Res 2010; 44(5): 479-496. doi: 10.3109/10715761003667554.

17. Ruiz-Ruiz C, Munõz-Pinedo C, López-Rivas A. Interferon- $\gamma$ treatment elevates caspase- 8 expression and sensitizes human breast tumor cells to a death receptor-induced mitochondria-operated apoptotic program. Cancer Res 2000; 60(20): 5673-5680. 